\title{
Comparing Emissions Mitigation Efforts across Countries
}

\section{Citation}

Aldy, Joseph E., William A. Pizer, and Keigo Akimoto. 2015. Comparing Emissions Mitigation Efforts Across Countries (June 23, 2015). Resources for the Future Discussion Paper 15-32.

\section{Published Version}

http://www.rff.org/files/document/file/RFF-DP-15-32.pdf

\section{Permanent link}

http://nrs.harvard.edu/urn-3:HUL.InstRepos:30203383

\section{Terms of Use}

This article was downloaded from Harvard University's DASH repository, and is made available under the terms and conditions applicable to Other Posted Material, as set forth at http:// nrs.harvard.edu/urn-3:HUL.InstRepos:dash.current.terms-of-use\#LAA

\section{Share Your Story}

The Harvard community has made this article openly available.

Please share how this access benefits you. Submit a story.

\section{Accessibility}


Comparing Emissions Mitigation Efforts across Countries

September 30, 2015 Draft

Joseph E. Aldy
Harvard University
Resources for the Future
National Bureau of Economic
$\quad$ Research
Center for Strategic and
$\quad$ International Studies
79 JFK Street, Mailbox 57
Cambridge, MA 02138
USA joseph aldy@hks.harvard.edu
+1617-496-7213

\author{
William A. Pizer \\ Duke University \\ Resources for the Future \\ National Bureau of Economic \\ Research \\ Center for Global Development \\ Box 90239 \\ Durham, NC 27708 \\ USA \\ billy.pizer@duke.edu \\ +1 919-613-9286
}

\author{
Keigo Akimoto \\ Research Institute of Innovative \\ Technology for the Earth \\ University of Tokyo \\ 9-2 Kizugawadi, Kizugawa-Shi \\ Kyoto 619-0292 \\ Japan \\ aki@rite.or.jp \\ $+81774-75-2300$
}

Abstract: A natural outcome of the emerging pledge and review approach to international climate change policy is the interest in comparing mitigation effort among countries. Domestic publics and stakeholders will have an interest in knowing if peer countries are undertaking (or planning to undertake) comparable effort in mitigating their greenhouse gas emissions. Moreover, if the aggregate effort is considered inadequate in addressing the risks posed by climate change, then this will likely prompt a broader interest in identifying those countries where greater effort is arguably warranted based on comparison with their peers. Both assessments require metrics of effort and comparisons among countries. We propose a framework for such an exercise, drawing from a set of principles for designing and implementing informative metrics. We present a template for organizing metrics on mitigation effort, for both ex ante and ex post review. We also provide preliminary assessments of effort along emissions, price, and cost metrics for post-2020 climate policy contributions by China, the European Union, Russia, and the United States. We close with a discussion of the role of academics and civil society in promoting transparency and facilitating the evaluation and comparison of effort.

Policy Relevance Statement: Our paper presents a framework for the review of intended nationally determined contributions and the ex post review of contributions under the UNFCCC negotiations. We provide an illustration of this framework with an energy-economic model. Our work focuses on how countries may use the review to compare mitigation effort - planned under INDCs and delivered by implementation of the pledged contributions - to address concerns about equity, efficiency, competitiveness, and the stability of any agreement that arise in international negotiations.

Keywords: emission mitigation, international environmental agreements, modeling analysis, reciprocity, policy surveillance, intended nationally determined contributions

JEL Codes: Q54, Q58, F55

Acknowledgments: We have benefitted from comments on our work at the 2014 Lima UN climate change talks, a seminar at Resources for the Future, as well as from Brian Flannery, Clayton Munnings, Ray Kopp, Mits Yamaguchi, and three referees.

Funding: This work was supported by the Research Institute of Innovative Technology for the Earth. 


\section{Comparing Emissions Mitigation Efforts across Countries}

\section{Introduction}

Free riding is the primary threat to any international climate change treaty. Any country considering taking domestic actions to mitigate its greenhouse gas emissions has a legitimate concern that other countries will not undertake similar actions to contribute to the global public good of a stable climate. Since the costs of mitigating emissions are local while the benefits are global, the prospect of free riding weakens incentives for countries to take serious actions against climate change. This is especially the case within the current multilateral "pledge and review" framework in which commitments are effectively self-enforced.

If one country is proactive in its emissions mitigation policy, then it will enjoy fewer benefits if other countries respond by free-riding. Moreover, absent such reciprocal action, unilateral mitigation efforts increase the prospect of adverse competitiveness impacts, which could raise the costs borne by the proactive country and enable domestic opposition to climate policy. In light of these factors undermining international cooperation and meaningful mitigation efforts, a system of transparency and comparability of efforts appears necessary for a successful international climate agreement premised on pledge and review (Schelling 1997; Pizer 2007; Aldy 2014).

The review and comparison of mitigation efforts can play an important role at the pledging stage. A review mechanism could estimate the environmental and economic impacts of countries' goals and domestic actions intended to serve as the countries' contribution to mitigation emissions. These ex ante reviews could identify those contributions that appear outof-step with other countries' contributions. This could facilitate peer pressure and stakeholder pressure for a country to ramp up its domestic program and/or goal. It could also highlight opportunities for potential learning from the variation in countries' mitigation programs.

The review and comparison of mitigation efforts can also play an important role in evaluating outcomes and performance. The review could ascertain if countries delivered on their pledged contributions. Ex post review could also illustrate how realized mitigation efforts compare across countries. The former can drive credibility in the iterative pledge and review 
process and the latter can enhance trust that countries are making fair contributions to mitigation emissions.

Given the complexity and heterogeneity in countries' pledges (the intended nationally determined contributions, or INDCS), a set of objective metrics can provide a common basis for characterizing countries' mitigation efforts in both ex ante and ex post reviews. Such metrics can enable an apples-to-apples comparison of countries' mitigation programs. We do not presume, however, that all countries should have equivalent values for these metrics. In light of the variation in national responsibility and capability (such as per capita emissions and per capita income), differences in value judgments, and country-specific domestic political considerations, countries will each employ its own normative framework when assessing its own and other countries' contributions. Indeed, this is reflected in the many interpretations of the UN Framework Convention on Climate Change's (UNFCCC) principle of common but differentiated responsibilities and respective capabilities in international negotiations. Building on the work in Aldy and Pizer (2014), our intention is to provide a common data and analytic framework that can measure mitigation efforts and facilitate the apples-to-apples comparisons, which can inform subsequent normative assessments.

In the next section we review the role of comparability in the history of the UN climate negotiations. We describe an array of metrics and discuss some of their advantages and shortcomings. We also consider potential facilitative and normative frameworks for using these metrics. We then employ the DNE21+ model to illustrate a suite of metrics for China, the EU, Russia, and the US based on their INDCs. We close with our suggestions for additional work, considerations for the design of reviews, and opportunities for academics and civil society to inform review processes.

\section{UNFCCC Processes and Comparability of Efforts}

The concept of comparable efforts has evolved over the past several decades in international climate change negotiations. The 1992 UNFCCC and the 1997 Kyoto Protocol set emissions targets for developed countries and established the first notion of a metric to compare effort: quantitative emissions limits relative to 1990 . The outcome also provided a 
normative example of differentiation, as the Kyoto negotiators agreed to individual country targets that ranged from $-8 \%$ to $+10 \%$ relative to their 1990 levels. Ex post, this turned out to be a simplistic and potentially misleading approach. For example, Russia's emissions have remained well below 1990 levels since the Kyoto Conference due to the state of its economy, not a broad and effective emissions mitigation program.

Independent, scholarly analysis of mitigation efforts have focused on a richer set of measures. For example, the Stanford Energy Modeling Forum organized thirteen modeling teams to evaluate the Kyoto Protocol (Weyant and Hill 1999). Through this exercise, the modelers estimated several metrics, including carbon prices (dollars per ton of carbon dioxide, $\mathrm{CO}_{2}$ ) and abatement costs (as a percentage of GDP). Emissions levels were implicit in the Kyoto commitments, and the modeling teams' forecasts of business-as-usual emissions would permit an estimate of emissions abatement. Finally, estimated emissions intensities $\left(\mathrm{CO}_{2}\right.$ per unit of GDP) could be produced from the model outputs.

The term "comparability of effort" first emerged explicitly in the 2007 Bali Action Plan, which noted that the concept should guide consideration of developed countries' mitigation efforts. At the 2009 Copenhagen Conference, the EU and Japan each announced conditional commitments: a willingness to implement more ambitious domestic emissions targets if other developed countries committed to comparable reductions. Different countries, however, held different perspectives on how to measure and compare efforts, and whether to compare efforts among only developed countries or to include emerging economies, such as China and India. To promote the transparency of these mitigation pledges and facilitate a better understanding of effort, the 2009 Copenhagen Accord and the 2010 Cancun Agreements included "international consultations and analysis" and "international assessment and review" - review mechanisms comprising reporting, technical analysis, and consultation with other parties. The first of these consultations took place in 2014.

Complementing (and preceding) these UNFCCC reviews, a number of analysts assessed and compared the Copenhagen Accord pledges. For example, McKibbin et al. (2011) compare emissions, emissions abatement, carbon prices, and costs (percentage changes in GDP and 
percentage changes in consumption). In an OECD report, Dellink and Corfee-Morlot (2010) estimate emissions reductions, relative to both baseline and a base year, carbon prices, fiscal revenues (assuming a carbon tax), energy-intensive industrial production, and costs (changes in GDP and changes in household income). Houser (2010) also compares countries' emissions reductions under Copenhagen Accord mitigation pledges. ${ }^{1}$

The emerging international climate architecture reflected in the 2014 Lima climate talks further advanced the concept of pledge and review, building on the Copenhagen model. Many countries have tabled their intended nationally determined contributions in the negotiations over the course of 2015. Through this pledging process, the Lima Call for Climate Action notes that countries may submit additional information, including data, analysis, methods, and descriptions of implementation timeframes and planning processes that may promote the transparency and credibility of countries' INDCs.

This evolution illustrates how analysis can inform the implementation of the concept of comparability of mitigation efforts. In the 2009 Copenhagen Accord and in the submitted INDCs leading up to the 2015 Paris talks, countries' emissions mitigation pledges take many different forms: targets versus 1990 or 2005 base year emissions, percentage improvements in the $\mathrm{CO}_{2}{ }^{-}$ to-GDP ratio, percentage abatement versus an emissions forecast, an emissions peak year, renewable power goals, energy efficiency goals, etc. The pledges themselves do not facilitate comparison. Even if countries report common metrics characterizing their own pledges, they will employ different methods and assumptions and thus, for a given metric, countries will produce "apples and oranges." ${ }^{2}$ In this pledge and review architecture, negotiators, stakeholders, and the general public would benefit from a harmonized characterization of the efforts that countries propose to undertake in the international negotiations. Economic data and analysis can play an important role in providing this information, enhance the credibility of countries' pledges, and lead to stronger pledges in the future. Indeed, an informed

\footnotetext{
${ }^{1}$ A number of groups began discussing the pledges leading up to the 2015 Paris talks (e.g., Climate Action Tracker 2014; WRI 2015).

${ }^{2}$ The variation in data and methods employed in country-produced national communications preclude a meaningful comparison across countries at a point time (i.e., across a given vintage of national communications) (Thompson 2006).
} 
consideration of countries' mitigation pledges cannot occur in the absence of an assessment of the estimated emissions levels, emissions abatement, carbon and energy price effects, and costs of implementation.

\section{Description of Comparability Metrics}

Aldy and Pizer (2014) have compiled a set of metrics for measuring effort in terms of emissions, prices, and costs. Emissions correspond to physical outcome measures that are most closely tied to the environmental problem and most commonly employed as the form of a mitigation commitment. Prices on carbon and of energy reflect market signals designed through mitigation policies and can represent the marginal incentives to reduce energy use and $\mathrm{CO}_{2}$ emissions. Cost metrics include measures of economic resources diverted away from current consumption and nonclimate investment.

These are evaluated in the context of three principles for desirable metrics. First, an ideal metric should be comprehensive and thereby characterize a country's entire effort to achieve its mitigation commitment. Second, a metric should be measurable and replicable. That is, different analysts making calculations of the metric should come up with the same values. Third, metrics should be universal-they should be available for a wide range of parties making mitigation commitments.

First, let us consider emissions-based metrics. Annual measures of $\mathrm{CO}_{2}$ emissions and total greenhouse gas emissions are now available for most countries (e.g., see World Resources CAIT database), even if less than a quarter of UNFCCC parties officially report their annual emission inventories. The use of market-based mechanisms-including international emissions trading and transfers of offset credits-requires additional accounting regarding a country's net position in international trades.

The metric used in the Kyoto context is a country's emissions relative to a 1990 base year. ${ }^{3}$ Unless countries are on similar trajectories prior to the commitment, however, emissions levels relative to a base year will not comprehensively represent mitigation efforts. In fact, it

\footnotetext{
${ }^{3}$ Note that a number of eastern European and former Soviet republics listed in Annex B of the Kyoto Protocol could employ alternative base years for their commitments.
} 
may have little to do with such efforts, as noted with respect to the economic transition in Russia and other former Soviet republics in the 1990s. Emissions trends vary from country to country for a number of reasons beyond government emission mitigation policies. While the issue in the former Soviet republics may have been declining economic activity, the larger issue is often concern about rising economic activity, particularly in emerging economies.

To avoid penalizing economic growth, some countries prefer a metric reflecting reductions in emissions intensity $\left(\mathrm{CO}_{2}\right.$ per GDP) rather than emissions alone. ${ }^{4}$ In the run-up to the 2009 Copenhagen talks, China and India each proposed to reduce the ratio of their emissions to GDP, and China has included such an emission intensity goal as part of its 2015 INDC. Such metrics can ensure that a country is not penalized as a climate laggard simply because of faster economic growth nor rewarded simply because of economic decline. An emissions intensity metric suffers from two challenges as a measure of effort: growing countries often experience declining emissions intensity due to structural and technological change; and, emissions intensity targets often become more stringent if a country's growth is slower than expected and less stringent if it is faster than expected (Aldy 2004; Newell and Pizer 2008).

Some developing countries have focused on emissions goals specified as percentage reductions from a forecast level in a future year. For example, Brazil, Indonesia, and Korea pledged emissions mitigation goals of this form through the Copenhagen Accord, and Colombia, Indonesia, and Korea have incorporated emission reductions from a forecast baseline emission trajectory in their 2015 INDCs. In theory, by capturing the emissions reduction directly associated with a mitigation program, such a metric represents a more comprehensive indicator of mitigation efforts than emissions levels relative to a historic base year or scaled by GDP. In practice, however, such a metric requires subjective judgments, on which expert opinions will differ, to calculate the baseline forecasts that define the emissions goals. Forecasts could be gamed to make a target appear more ambitious than it will likely be in practice.

\footnotetext{
${ }^{4}$ We do not discuss emissions per capita as a measure of effort. Emissions per capita has been discussed as an ethical basis for allocating emission mitigation responsibility. We therefore include it in our discussion of how one might benchmark appropriate effort levels across countries.
} 
Constructing a baseline also raises conceptual questions. Do existing or previously announced mitigation policies go into baseline forecasts? Put another way: how far back in time should a definition of effort go in order to make comparisons across countries, recognizing early actors but not overly penalizing laggards?

In contrast, an observed carbon price bears a direct connection to effort, as it measures the marginal incentive levied through a country's mitigation policies. Comparing carbon prices across countries measures the degree to which each country is creating incentives for mitigation efforts. Since countries implement domestic carbon prices in their local currencies, comparisons will require the use of (and raise questions as to the appropriate) currency exchange rates. ${ }^{5}$ The volatility in carbon prices derived from cap-and-trade programs may also raise questions about the timing and frequency - and accuracy - of price comparisons. Explicit carbon prices may not comprehensively reflect mitigation effort, because they may fail to account for the effect of nonprice policies-such as efficiency and renewable mandates. A country may also undermine the effectiveness of the carbon price through fiscal cushioning adjusting taxes downward for firms covered by the carbon price (Wiener 1999). Alternatively, one could consider implicit (or effective) carbon prices that estimate the average cost of abatement associated with a specific climate policy or collection of policies (OECD 2013). Such implicit prices have the advantage of potentially being applied more broadly but the disadvantage of not being directly observed (i.e., produced by model simulations).

Energy prices are transparent and measurable with high frequency. Comparison of the user prices of primary energy and final energy products permits a net assessment of all pricebased policies, including carbon pricing, and thus can mitigate concerns about fiscal cushioning. Energy prices would still fail to capture the effects of nonprice policies that mitigate emissions without influencing energy prices (which explains why the latter often attract political support). For countries pursuing such policies to a significant degree, energy prices could be a poor measure of effort. Moreover, not all energy price differences represent policy choices, but

\footnotetext{
${ }^{5}$ This is also relevant for comparing $\mathrm{CO}_{2}$-to-GDP intensity levels.
} 
instead, prices may reflect differences in resource endowments, transportation constraints, or other shifts in supply and demand.

The mitigation costs of any domestic climate policy are typically most closely aligned with economists' notion of mitigation effort. Ideally, such a metric captures the entirety of resources otherwise available for private consumption and citizen well-being that are diverted toward the public good of mitigating climate change. Thus, it is appealing in terms of being comprehensive. Expressed as a share of national income, or per capita, it could be scaled to be comparable across countries of different sizes. A metric to compare efforts based on costs could promote confidence that the international efforts are fair by ensuring that comparable countries bear comparable costs resulting from their actions. Coupled with information about emissions reductions, it could also highlight the potential advantages of some policies (those that reduce more emissions with lower mitigation costs) over others. An assessment of policy costs alone runs the risk highlighting inefficient polices, so it may be appropriate to also calculate the least-cost alternative to achieve the same reductions. Estimating costs, in any case, requires economic assumptions and detailed modeling frameworks for evaluating economic changes in specific sectors and national economies.

Viewed alone, none of the six metrics described here does well against all three principles. Emissions levels and intensity do particularly poorly in terms of comprehensiveness, as many factors can influence emissions that are unrelated to mitigation policies. Carbon prices do well on effort per abated ton, but actual effort then depends on total tons of abatement. Observed carbon prices are available only where emissions trading or taxes are applied. Energy prices capture the market signals being sent for low-carbon investment and behavioral change but fail to reflect nonmarket policies. Emissions abatement and abatement costs are jointly the metrics that probably best represent effort, but they are the most difficult to measure, requiring sophisticated modeling tools for implementation. Credible differences in opinion on modeling assumptions could produce different results for abatement and costs, raising questions about replicability. Further, few modeling tools exist to address jurisdictions outside of the largest economies. 
Given the absence of a single, ideal metric and the complexity of assessing mitigation effort, we suggest using a suite of metrics to compare effort. Just as an analyst may rely on a set of metrics for evaluating human development or a set of metrics for characterizing a nation's macroeconomy, we believe that a portfolio of mitigation effort metrics could best characterize mitigation.

\section{A Template for Comparing INDCs and Potential Uses}

To organize the metrics to facilitate the evaluation of comparable efforts, we have developed a template, presented in Table 1. This framework identifies the data and analysis needs for constructing metrics to evaluate countries' mitigation pledges. Each column represents a major economy, with a brief description of a country's mitigation program in the first row. The left half of the table focuses on the ex ante analysis of comparability of efforts. For example, this could organize information on the intended nationally determined contributions announced by countries in the lead-up to the 2015 Paris talks (and in subsequent rounds of negotiations). The first row of the table would list briefly each country's INDC, and the subsequent rows would depict the estimated measure for each of the metrics. As the template shows, some of the metrics will be directly observed, others will require a baseline forecast, and still others will require modeling analyses.

The right half of the table presents a template for an ex post analysis, such as the "review" in the pledge and review regime in the current international climate negotiations. In this case, the summary of a country's climate program is the implemented contribution, rather than what it intended to do under the INDC. Some of the metrics that are modeled in the ex ante review would be directly observed in the ex post review. Nonetheless, a number of metrics will require counterfactual forecasts and economic modeling even in an ex post exercise, such as baseline emissions and economic activity. So long as the review of efforts addresses measures beyond physical emissions outputs or observed market prices, economic tools will need to be employed to quantify efforts in a manner that will permit comparisons. Standard economic tools may be employed even for observed metrics, such as aggregating observed energy prices over various fuels over time, to construct a summary of prices. 
Economic tools may also serve to illustrate the impacts of interactions across countries from the implementation of each country's individual INDCs.

Table 1. Information Sources for Comparability Metrics

The construction of metrics is a positive exercise. How policymakers, stakeholders, and the public interpret these metrics is a normative exercise. What is the benchmark or threshold for deciding whether a country's mitigation efforts are characterized as "fair" and "satisfactory?" Let us suggest three ways for addressing this question.

First, one could simply rank countries on each quantitative metric. This would identify those countries that are high, or low, on a metric and illustrate proximity of any pair of countries. Countries could be compared based on each individual metric or on a composite suite of measures, which would require an algorithm for integrating information from the various metrics, perhaps akin to implementation of the human development index. This approach would not answer the question of whether a country's performance is satisfactory, but it would provide a relative comparison.

Second, countries can be arranged or self-associate into peer groups for relative comparisons that reflects the significant variation in countries' emissions contribution and capacity to mitigate. Peer groups could reflect existing groupings of countries, such as UN regional groups or the Major Economies Forum on Energy and Climate (MEF). Alternatively, peer groups could be identified through measures associated with responsibility and capability in the climate talks, such as historic cumulative emissions, per capita emissions, and per capita income.

To illustrate this peer group approach, consider the MEF. The international community might agree on several official metrics, or the MEF countries might themselves select a set of metrics to compare efforts. The MEF countries could then voluntarily agree to present data and analysis regarding their future emissions commitments in order to produce these agreed metrics and to demonstrate feasibility and applicability of such a process. The MEF countries could further explore options of benchmarking and interpreting metrics in light of each country's national circumstances. 
These two approaches do not directly answer the question of whether a given country is undertaking "satisfactory" effort in a global sense. The first approach simply provides inputs that a country (or stakeholder) can use in its own normative framework for evaluating fairness. The second approach is premised on the concept that peer countries should undertake comparable efforts. This is useful in identifying leaders and laggards among peer countries, but provides no guidance about whether a country's effort is appropriate relative to a global objective. In contrast, a third approach could establish normative, absolute benchmarks for countries' contributions. Such benchmarks could be derived directly from a negotiated emissions commitment such as the Kyoto Protocol. Under the Kyoto Protocol, countries had individualized targets ranging from $-8 \%$ (most European countries) to $+10 \%$ (Iceland) relative to 1990 levels, presumably reflecting their particular circumstances or capacity. This differentiation was negotiated and collectively approved by negotiators and thus could be interpreted as "fair" based on political revealed preference. The lack of negotiation and collective approval of pledged contributions in the current framework precludes such an approach.

An alternative to negotiated, ad hoc adjustments is to develop a formulaic approach to address the question of what countries "should" do, i.e., how to allocate the burden of efforts (e.g., Höhne et al. 2014; Bosetti and Frankel 2012; den Elzen et al. 2006; Groenenberg et al. 2004; Gupta 2007; Hof and den Elzen 2010; Höhne et al. 2006; Michaelowa et al. 2005). ${ }^{6}$ Despite the appealing logic of such an approach, it seems unlikely that negotiators will agree on a set of official country-level benchmarks in the current framework organized around sovereign, voluntary pledges of emissions contributions. Such approaches will continue to play a role in unofficial judgments by governments as well as various stakeholders and academics. We highlight a few issues that merit consideration in this discussion.

First, one could design a benchmark associated with the UNFCCC's long-term objective. For example, the Copenhagen Accord and Cancun Agreements identify a long-term objective to limit warming to no more than $2^{\circ} \mathrm{C}$, which could be linked (with admitted uncertainty) to a trajectory of cumulative global emissions levels, and subsequently decomposed into country-

\footnotetext{
${ }^{6}$ Related to these papers, the Government of South Africa proposed an equity reference framework for INDCs in the lead-up to the 2015 Paris climate negotiations.
} 
specific emission budgets (e.g., Tavoni et al. 2015). ${ }^{7}$ Translating a $2^{\circ} \mathrm{C}$ objective into national emissions benchmarks requires a framework for determining national emission budgets. With a number of countries, including Switzerland and the US, claiming that their 2015 INDCs are consistent with a $2^{\circ} \mathrm{C}$ objective, it would useful to study and understand the budget frameworks implicit in their INDCs.

As an alternative to emission-based benchmarks, one could design a price-based benchmark. For example, the social cost of carbon (SCC), which has been used by a few countries in informing their domestic emissions mitigation programs (Pizer et al. 2014), could work well for evaluating and comparing carbon pricing metrics across countries. The challenges with a SCC benchmark include reaching agreement on a SCC estimate and deciding which countries should have carbon prices consistent with the SCC (e.g., should low-income countries be exempt from this benchmark?).

Finally, benchmarks could be established as a function of a country's per capita emissions, historic cumulative emissions, per capita income, or other measures of national responsibility and capabilities. Such functions could be used to translate an aggregate objective into national benchmarks, or to translate and compare metrics across disparate countries. This goes far beyond the simpler idea that peer groups of countries could be expected to deliver comparable mitigation effort.

Whether ad hoc or formulaic, any attempt to label national efforts as satisfactory or not requires addressing a host of thorny questions. These range from adjustments for population growth, wealth, and fossil resources, to judgments about past actions and the weight placed on new efforts. The difficulty of these questions-and the strong likelihood that official, negotiated answers may not be forthcoming - suggests a likely continuing role for independent, albeit unofficial, work in this area.

5. Illustration of Comparability of Efforts Framework

\footnotetext{
${ }^{7}$ Tavoni et al (2015) provide an illustration of this kind of cumulative budgets analysis.
} 
To provide an initial illustration of the comparability of efforts, we focus on the announcements for post-2020 mitigation contributions by several of the largest economies in the world, including China, the EU, Russia, and the US. The top of Table 2 summarizes their respective INDCs.

We have employed the RITE DNE21+ model in undertaking this assessment. Given a specific policy (e.g., a set of INDCs), DNE21+, a linear programming model, searches for the solution that minimizes energy system costs globally (Akimoto et al. 2008, 2012; RITE 2015). The model runs in a combination of 5- and 10-year time steps over 2000-2050 with the world divided into 54 regions. The model includes 200+ energy technologies in a bottom-up modeling scheme, with an explicit characterization of existing facility vintages. It also includes interregional trade in coal, crude oil, natural gas, ethanol, hydrogen, electricity, and $\mathrm{CO}_{2}$ allowances. While this model is quite rich in how it captures the energy economy, it does not cover non-energy related greenhouse gas emissions or land use change. ${ }^{8}$ For some countries, such as those with significant agriculture-related methane emissions or reforestation activities, we recommend alternative analytic frameworks. See RITE (2015) for more details.

Historical greenhouse gas emissions are derived from the UNFCCC data where possible (US, EU, and Russia) and otherwise from IEA statistics (China). Emission and GDP are taken from the DNE21+ model for consistency with the cost estimations. The growth rates of real GDP between 2010 and 2030 are 1.2\%, 5.3\%, and 6.6\% per year for the EU, Russia, and China, and that between 2010 and 2025 for the US is $2.4 \%$ per year. These are nearly consistent with the reference forecasts published in the International Energy Outlook (EIA 2013) and the World Energy Outlook of (IEA 2013). Baseline emission forecasts are comparable to other energyeconomy and integrated assessment models (see Kriegler et al 2014). Note that these baselines exclude explicit, existing climate policies. This differs from the approach of the EIA and IEA that include such policies (e.g., the EU ETS) in their forecasts. In contrast to EIA and IEA forecasts, our approach will give credit to countries for those existing carbon pricing policies when measuring emission changes and costs against baseline forecasts.

\footnotetext{
${ }^{8}$ For these modeling simulations, we are thus illustrating this framework by applying the countries' INDCs only to fossil fuel $\mathrm{CO}_{2}$ emissions.
} 
Table 2 presents the preliminary results for the US, the EU, Russia, and China based on their INDCs. The US INDC is extrapolated to 2030 based on a reduction of $83 \%$ below 2005 levels by 2050, consistent with past US government policy statements. The EU and Russian targets are similarly interpolated to 2025. For the China INDC, we have modeled the reduction in emission intensity and estimate that it will deliver on the 2030 emissions peaking objective. In each of the four cases, the model solves for the carbon price that would deliver the 2025 or 2030 emissions level or emissions intensity (or midpoint of the range) established in the INDCs.

The table presents metrics for emissions relative to various base years, emissions intensity in levels and changes over time, changes in emissions relative to emissions forecasts, estimated marginal abatement costs, and total abatement costs scaled by GDP. As suggested earlier, the choice of base year for emissions and emissions intensity matters significantly in the levels and rankings among countries. A 1990 base year yields much larger percentage reductions for the EU and Russia contributions, relative to the US and China, than a 2005 base year. Changing the base year for Russia results in a different sign, indicating that its contribution implies emissions growth of $14 \%$ from its 2005 level, while it would be a $28 \%$ reduction from its 1990 level. The US (-27\%) and the EU (-35\%) have relatively similar percentage reductions from their 2005 emissions. The US and EU have similar reductions in emissions intensity through 2030 as well; China and Russia both have even faster declines. As noted earlier, reductions versus $\mathrm{BAU}$ is perhaps the most comprehensive metric among emission measures. Here, China and Russia show relatively small reductions from what their emissions would be otherwise, while the US and the EU show larger, also comparable reductions (-36\% and $-42 \%)$.

The prices of energy and the estimated mitigation costs necessary to deliver on the INDCs vary across these four countries/regions. The US and the EU targets are associated with meaningful carbon prices (marginal abatement costs) and costs as a share of GDP, while these are approximately zero for China and Russia. This is consistent with the comparison of reductions versus baseline forecasts. Meanwhile, the substantial variation in energy prices reflects the continuation of existing subsidies in some countries (e.g., Russia), variation in excise taxes (e.g., contrast the US and EU), as well as access to low-cost energy (e.g., US natural gas). 
The nature of this illustration reflects the view that these four countries/regions are peers and thus merit direct comparison. In terms of their economic size, annual greenhouse gas emissions, and potential for diplomatic leadership in the climate talks, this could be a reasonable conclusion. ${ }^{9}$ The differences in per capita income and wealth with the US and EU, on one hand, and China and Russia, on the other, may suggest alternative benchmarks when comparing effort among them.

Regardless of whether China and Russia are considered peers to the US and EU, many stakeholders would expect their mitigation efforts to be sufficiently ambitious to impose real costs. Among the metrics we develop, we have pointed to cost as the most comprehensive effort metric from an economist's perspective. It represents resources given up by society for climate change mitigation. In this regard, the US and the EU propose to do more (the EU twice as much as the US) and China and Russia considerably less (the numbers vanish in the model's measurement error). The uncertainty in forecasting BAU, especially with respect to Chinese economic growth and emission intensity improvements, indicates the potential value in extensive sensitivity analysis, which could find more mitigation effort and higher costs in China under its INDC. The primary objective of our analysis is NOT to provide a definitive conclusion on the comparability of effort among these countries but to provide objective information that can be processed by and subsequently guide decisions by governments and the advocacy of stakeholders.

Table 2. The preliminary INDCs of the US, the EU, Russia, and China by different metrics

\section{Conclusions}

Metrics to compare climate change actions across countries are increasingly relevant as we transition to a program of periodic, unilateral pledges of domestic actions and policies within international negotiations. The emerging architecture calls for countries to state their emission mitigation contributions and encourages countries to provide detailed information to facilitate the understanding of the contributions. Regardless of whether the UN climate

\footnotetext{
${ }^{9}$ The recent bilateral China-US climate agreements also suggest that they view each other as partners.
} 
negotiations result in an agreement on a formal review mechanism, individual countries and stakeholders will review the pledged contributions to inform their judgments about the adequacy and fairness of the INDCs, and then react accordingly. This reaction could include enhanced contributions in the formal negotiations or increased domestic activity beyond the current pledge. Moreover, the review process may enable countries to learn how to improve the design of their own policy response over time, promoting cost-effectiveness and environmental ambition (Aldy 2013).

In considering metrics for comparability, several important distinctions emerge. Some metrics, such as total emissions and explicit emissions prices, are relatively easy to observe and measure but may not be closely related to effort and policy implementation. The concepts that are closer to effort-emission reductions, implicit prices, and costs-are harder to observe and measure directly. These will require explicit modeling tools, leading to more subjective and possibly divergent estimates. We have presented one set of preliminary modeling analyses to illustrate the application of this framework for China, the EU, Russia, and the US.

Developing metrics for assessing comparability of efforts, compiling data and related analysis to estimate metrics, and providing feedback on the assessments will require a serious, transparent, and legitimate process (Aldy and Stavins 2012; Aldy 2014). Independent researchers can fill the gap until agreement on an official policy surveillance mechanism is reached. Given existing data collection by international organizations, an array of easily available metrics could be developed to facilitate comparisons in the near term. Unofficial but independent expert analysis could synthesize these data to construct some of the more challenging but informative metrics. In turn, stakeholders and other users could provide feedback on the feasibility, integrity, and precision of various metrics to enable further refinement and enhance their value to the negotiations. 


\section{References}

Akimoto, Keigo, Fuminori Sano, Takashi Homma, Kenichi Wada, Miyuki Nagashima, and Junichiro Oda. 2012. Comparison of Marginal Abatement Cost Curves for 2020 and 2030: Longer Perspectives for Effective Global GHG Emission Reductions. Sustainability Science 7: 157-68.

Akimoto, Keigo, Fuminori Sano, Junichiro Oda, Takashi Homma, Ullash Kumar Rout, and Toshimasa Tomoda. 2008. Global Emission Reductions through a Sectoral Intensity Target Scheme. Climate Policy 8: S46-S59.

Aldy, Joseph E. 2004. Saving the Planet Cost-Effectively: The Role of Economic Analysis in Climate Change Mitigation Policy. In Painting the White House Green: Rationalizing Environmental Policy inside the Executive Office of the President, edited by Randall Lutter and Jason F. Shogren, 89-118. Washington, DC: Resources for the Future Press.

- - - 2013. Designing a Bretton Woods Institution to Address Global Climate Change. In Handbook of Energy and Climate Change, edited by Roger Fouquet, 352-374. Cheltenham, UK: Edward Elgar.

- - - 2014. The Crucial Role of Policy Surveillance in International Climate Policy. Climatic Change 126: 279-92.

Aldy, Joseph E., and William A. Pizer. 2014. Comparability of Effort in International Climate Policy. Discussion paper 14-62. Cambridge, MA: Harvard Project on Climate Agreements. Aldy, Joseph E., and Robert N. Stavins. 2012. Climate Negotiators Create an Opportunity for Scholars. Science 337: 1043-44.

Bosetti, Valentina, and Jeffrey Frankel. 2012. Politically Feasible Emission Targets to Attain 460ppm $\mathrm{CO}_{2}$ Concentrations. Review of Environmental Economics and Policy 6: 86-109.

Climate Action Tracker. 2014. http://www.climateactiontracker.org.

Dellink, Rob, and Jan Corfee-Morlot. 2010. Costs and Effectiveness of the Copenhagen Pledges: Assessing Global Greenhouse Gas Emissions Targets and Actions for 2020. Organisation for Economic Co-operation and Development (OECD). http://www.oecd.org/environment/cc/45441364.pdf. 
den Elzen, Michel G. J., Marcel Berk, Paul Lucas, Patrick Criqui, and Alban Kitous. 2006. MultiStage: A Rule-Based Evolution of Future Commitments under the Climate Change Convention. International Environmental Agreements: Politics, Law and Economics 6: 128.

Energy Information Administration. 2013. International Energy Outlook 2013. Washington: Department of Energy.

Groenenberg, Heleen, Kornelis Blok, and Jeroen van der Sluijs. 2004. Global Triptych: A BottomUp Approach for the Differentiation of Commitments under the Climate Convention. Climate Policy 4: 153-75.

Gupta, Joyeeta. 2007. Beyond Graduation and Deepening: Toward Cosmopolitan Scholarship. In Architectures for Agreement: Addressing Global Climate Change in the Post-Kyoto World, edited by Joseph E. Aldy and Robert N. Stavins, 116-30. Cambridge: Cambridge University Press.

Hof, Andries F., and Michel G. J. den Elzen. 2010. The Effect of Different Historical Emissions Datasets on Emission Targets of the Sectoral Mitigation Approach Triptych. Climate Policy 10: 684-704.

Höhne, Niklas, Michel den Elzen, and Donovan Escalante. 2014. Regional GHG Reduction Targets Based on Effort Sharing: A Comparison of Studies. Climate Policy 14: 122-147. Höhne, Niklas, Michel den Elzen, and Martin Weiss. 2006. Common but Differentiated Convergence (CDC): A New Conceptual Approach to Long-Term Climate Policy. Climate Policy 6: 181-99.

Houser, Trevor (2010). Copenhagen, the Accord, and the Way Forward. Petersen Institute for International Economics Policy Brief PB10-5. http://www.iie.com/publications/pb/pb10-05.pdf.

International Energy Agency. 2013. World Energy Outlook 2013. Paris: IEA.

Kriegler, Elmar, et al. (2014). The Role of Technology for Achieving Climate Policy Objectives: Overview of the EMF 27 Study on Global Technology and Climate Policy Strategies. Climatic Change 123: 353-367. 
McKibbin, Warwick J., Adele C. Morris, and Peter J. Wilcoxen. 2011. Comparing Climate Commitments: A Model-Based Analysis of the Copenhagen Accord. Climate Change Economics 2: 79-103.

Michaelowa, Axel, Sonja Butzengeiger, and Martina Jung. 2005. Graduation and Deepening: An Ambitious Post-2012 Climate Policy Scenario. International Environmental Agreements: Politics, Law and Economics 5: 25-46.

Newell, Richard N., and William A. Pizer. 2008. Indexed Regulation. Journal of Environmental Economics and Management 56: 221-33.

OECD (Organisation of Economic Co-operation and Development). 2013. Effective Carbon Prices. Paris: OECD.

Pizer, William A. 2007. Practical Global Climate Policy. In Architectures for Agreement: Addressing Global Climate Change in the Post-Kyoto World, edited by Joseph E. Aldy and Robert N. Stavins, 280-314. Cambridge: Cambridge University Press.

Pizer, William, Matthew Adler, Joseph Aldy, David Anthoff, Maureen Cropper, Kenneth Gillingham, Michael Greenstone, Brian Murray, Richard Newell, Richard Richels, Arden Rowell, Stephanie Waldhoff, and Jonathan Wiener. 2014. Using and Improving the Social Cost of Carbon. Science 346: 1181-82.

RITE (Research Institute of Innovative Technology for the Earth). 2015. RITE GHG Mitigation Assessment Model DNE21+. http://www.rite.or.jp/Japanese/labo/sysken/about-globalwarming/download-data/RITE GHGMitigationAssessmentModel 20150130.pdf.

Schelling, Thomas C. 1997. The Cost of Combating Global Warming. Foreign Affairs 76 (6): 8-14. Tavoni, Massimo et al. 2015. Post-2020 Climate Agreements in the Major Economies Assessed in Light of Global Models. Nature Climate Change 5: 116-126.

Thompson, Alexander. 2006. Management under Anarchy: The International Politics of Climate Change. Climatic Change 78: 7-29.

Weyant, John P., and Jennifer Hill. 1999. Introduction and Overview, in The Costs of the Kyoto Protocol: A Multi-model Evaluation, special issue, Energy Journal (May): vii-xliv.

Wiener, Jonathan. 1999. Global Environmental Regulation: Instrument Choice in Legal Context. Yale Law Journal 108: 677-800. 
WRI (World Resources Institute). 2015. CAIT Paris Contributions Map. http://cait.wri.org/indc/. 
Table 1. Information Sources for Comparability Metrics

\begin{tabular}{|c|c|c|c|c|}
\hline & \multicolumn{3}{|c|}{ Ex Ante Analysis } & Ex Post Analysis \\
\hline & US & EU & China & China \\
\hline INDC description & $\begin{array}{c}2005-26 \% \text { to }-28 \% \\
\text { by } 2025\end{array}$ & $\begin{array}{l}1990-40 \% \\
\text { by } 2030\end{array}$ & $\begin{array}{l}\text { Peaking by } 2030, \\
\mathrm{CO}_{2} / \text { GDP goal }\end{array}$ & Implemented Contributions (TBD) \\
\hline \multicolumn{5}{|l|}{ Emissions } \\
\hline vs. historical year & \multicolumn{2}{|c|}{$<$ directly observed $>$} & <requires modeling > & $<$ directly observed $>$ \\
\hline vs. future year BAU & \multicolumn{2}{|c|}{$<$ requires forecast $>$} & $<$ requires modeling $>$ & $<$ requires modeling $>$ \\
\hline Target year GHG/GDP & \multicolumn{2}{|c|}{$<$ requires forecast $>$} & $<$ requires modeling $>$ & $<$ directly observed > \\
\hline$\Delta$ (GHG/GDP) 2015-25 & \multicolumn{2}{|c|}{$<$ requires forecast $>$} & $<$ requires modeling $>$ & $<$ directly observed $>$ \\
\hline$\Delta$ (GHG/GDP) 2015-30 & \multicolumn{2}{|c|}{$<$ requires forecast $>$} & $<$ requires modeling $>$ & $<$ directly observed $>$ \\
\hline \multicolumn{5}{|l|}{ Price } \\
\hline $\mathrm{CO}_{2}$ & \multicolumn{2}{|c|}{ <requires modeling> } & <requires modeling > & $\begin{array}{l}\text { <carbon pricing: directly observed / } \\
\text { other policies: require modeling > }\end{array}$ \\
\hline Fossil energy & \multicolumn{2}{|c|}{ <requires modeling > } & <requires modeling > & $<$ directly observed $>$ \\
\hline Electricity & \multicolumn{2}{|c|}{$<$ requires modeling $>$} & $<$ requires modeling $>$ & $<$ directly observed $>$ \\
\hline \multicolumn{5}{|l|}{ Cost } \\
\hline$\$$ cost vs. BAU & \multicolumn{2}{|c|}{ <requires modeling > } & <requires modeling $>$ & $<$ requires modeling > \\
\hline$\$$ cost/GDP & \multicolumn{2}{|c|}{ <requires modeling> } & $<$ requires modeling $>$ & <requires modeling > \\
\hline
\end{tabular}


Table 2. Ex ante assessment of the INDCs of the US, the EU, China, and Russia

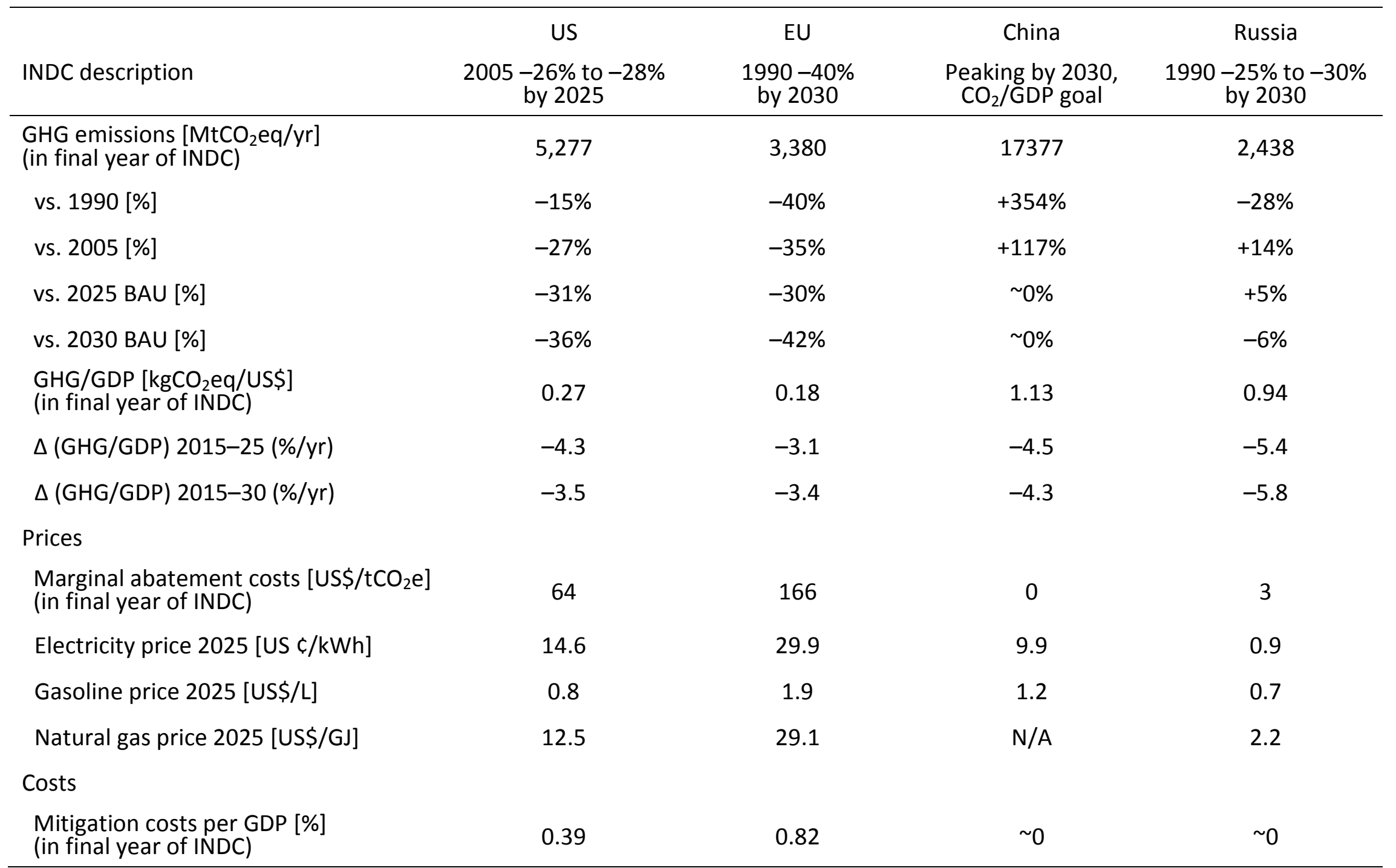

Note: BAU, business-as-usual, is the baseline scenario estimated in DNE21+. For the US, China, and Russia, we have employed the midpoint in their INDC range. 